\title{
Implications of partial immunity on the prospects for tuberculosis control by post-exposure interventions
}

${ }^{a}$ Instituto Gulbenkian de Ciencia, Apartado 14, 2781-901 Oeiras Cedex, Portugal

${ }^{\mathrm{b}}$ Faculdade de Ciências de Lisboa and Centro de Matemática e Aplicaçóes Fundamentais, Av. Prof. Gama Pinto 2, 1649-003 Lisboa, Portugal

${ }^{\mathrm{c}}$ Faculdade de Ciências e Tecnologia, Universidade Nova de Lisboa, Monte da Caparica, 2825-114 Caparica, Portugal

${ }^{\mathrm{d}}$ Ecology and Epidemiology Group, Department of Biological Sciences, University of Warwick, Coventry, CV4 7AL, UK

Received 4 October 2006; received in revised form 15 May 2007; accepted 6 June 2007

\begin{abstract}
One-third of the world population (approximately 2 billion individuals) is currently infected with Mycobacterium tuberculosis, the vast majority harboring a latent infection. As the risk of reactivation is around $10 \%$ in a lifetime, it follows that 200 million of these will eventually develop active pulmonary disease. Only therapeutic or post-exposure interventions can tame this vast reservoir of infection. Treatment of latent infections can reduce the risk of reactivation, and there is accumulating evidence that combination with postexposure vaccines can reduce the risk of reinfection. Here we develop mathematical models to explore the potential of these postexposure interventions to control tuberculosis on a global scale. Intensive programs targeting recent infections appear generally effective, but the benefit is potentially greater in intermediate prevalence scenarios. Extending these strategies to longer-term persistent infections appears more beneficial where prevalence is low. Finally, we consider that susceptibility to reinfection is altered by therapy, and explore its epidemiological consequences. When we assume that therapy reduces susceptibility to subsequent reinfection, catastrophic dynamics are observed. Thus, a bipolar outcome is obtained, where either small or large reductions in prevalence levels result, depending on the rate of detection and treatment of latent infections. By contrast, increased susceptibility after therapy may induce an increase in disease prevalence and does not lead to catastrophic dynamics. These potential outcomes are silent unless a widespread intervention is implemented.
\end{abstract}

(C) 2007 Elsevier Ltd. All rights reserved.

Keywords: Tuberculosis; Post-exposure interventions; Mathematical model; Reinfection threshold; Bistability

\section{Introduction}

Mycobacterium tuberculosis is transmitted by airborne particles and tuberculosis $(\mathrm{TB})$ infection results when the mycobacterium is deposited in the lungs of exposed persons. The infection is maintained in a latent form for a variable length of time. A small proportion of persons progress to active TB and become infectious within the first years of infection, while the remainder maintains a latent infection. Latent infections are asymptomatic and do not

\footnotetext{
*Corresponding author. Instituto Gulbenkian de Ciência, Apartado 14, 2781-901 Oeiras cedex, Portugal. Tel.: +351214464626; fax: +351214407970 .

E-mail address: ggomes@igc.gulbenkian.pt (M. Gabriela M. Gomes).
}

contribute to transmission, but may progress to active TB through either endogenous reactivation or exogenous reinfection (Small and Fujiwara, 2001; Styblo, 1978). The relative importance of these two mechanisms is subject to a long and intensive debate, but there is increasing consensus that it depends on the epidemiological context (Chiang and Riley, 2005; Gomes et al., 2004a; Vynnycky and Fine, 1997).

Three types of medical interventions are available to address TB: treatment to cure active $\mathrm{TB}$; vaccination to prevent infection; treatment of latent $\mathrm{TB}$ to prevent endogenous reactivation. While it is generally agreed that continued attention should be given to the treatment of active TB cases, there is no consensus about the use of 
1 preventive measures. The bacille Calmette-Guérin (BCG) is the only vaccine in current use against $\mathrm{TB}$, and its limitations are well recognized (Bloom and Fine, 1994). Preventive therapy is normally restricted to individuals with TB-HIV co-infection and contacts of tuberculosis patients. This control measure has $60-80 \%$ efficacy, and yet its impact on mortality and transmission in low- and middle-income countries is low, as compared to other available interventions (Borgdorff et al., 2002). Treatment of latent infections is not extended to the entire latent population because it requires that large doses of antibiotics are taken for long periods of time, which is costly and difficult to achieve (Bloom and McKinney, 1999). These caveats have motivated research into the development of therapeutic vaccines that could block reactivation and simultaneously reduce the risk of reinfection (Andries et al., 2005; Ha et al., 2003, 2005; Lowrie et al., 1999; Malowany et al., 2006; McShane et al., 2004). Here, we analyze different scenarios for post-exposure interventions by way of mathematical modeling.

Previous models addressing the potential impact of preand post-exposure interventions in tuberculosis dynamics have, either totally or partly, excluded reinfection (Lietman and Blower, 2000; Murphy et al., 2003; Ziv et al., 2001, 2004). However, recent studies show that reinfection is an important cause of pulmonary tuberculosis in regions of very high (van Rie et al., 1999), moderate (Caminero et al., 2001) and low (Bandera et al., 2001) incidence. The results presented here demonstrate that reinfection can dramatically change the outcome of post-exposure interventions.

Little is known about the immune mechanisms underlying protection against active TB and whether the same immune mechanisms mediate protection against both reactivation and reinfection. It is generally recognized that treatment of latent TB reduces the chances of reactivation. However, it is still unknown how treatment influences reinfection. Therefore, results will be presented for the three basic scenarios: (i) treatment of latent TB does not affect the risk of developing active TB upon reinfection; (ii) treatment reduces susceptibility to reinfection and (iii) treatment increases susceptibility to reinfection.

\section{Model description}

A mathematical model is posed, based on the assumption that all individuals are equally susceptible at birth and differentiate as they experience infection and in its case therapy. We specify five categories for the proportions of the population being: $(S)$ susceptible, who have never encountered the mycobacterium; $\left(L_{1}\right)$ early latent, representing individuals that were recently infected (typically within less than two years) and have not yet developed active TB; $(I)$ infected, who have active TB and are infectious; $\left(L_{2}\right)$ persistent latent, representing individuals who were infected and remain latent; $(R)$ recovered individuals who were previously infected and treated. As individuals experience infection, immunity and therapy, the proportions of the population in each category change, as represented by the diagram in Fig. 1 and described by the system of equations:

$$
\begin{aligned}
\frac{\mathrm{d} S}{\mathrm{~d} t} & =\mu-\Lambda S-\mu S, \\
\frac{\mathrm{d} L_{1}}{\mathrm{~d} t} & =\Lambda\left(S+\sigma L_{2}+\sigma_{R} R\right)-\left(\delta+\tau_{1}+\mu\right) L_{1}, \\
\frac{\mathrm{d} I}{\mathrm{~d} t} & =\phi \delta L_{1}+\omega L_{2}+\omega_{R} R-\left(\tau_{0}+\mu\right) I, \\
\frac{\mathrm{d} L_{2}}{\mathrm{~d} t} & =(1-\phi) \delta L_{1}-\sigma \Lambda L_{2}-\left(\omega+\tau_{2}+\mu\right) L_{2}, \\
\frac{\mathrm{d} R}{\mathrm{~d} t} & =\tau_{0} I+\tau_{1} L_{1}+\tau_{2} L_{2}-\sigma_{R} \Lambda R-\left(\omega_{R}+\mu\right) R .
\end{aligned}
$$

The parameters and their values used throughout the paper are described in Table 1. It should be noted that the state variables are written as fractions of the total population which is assumed to be constant, i.e. the rate of birth and death, $\mu$, are equal (corresponding to a mean life time of 70 years) and there are no disease-related deaths. As we will point out in Discussion, the results presented here do not depend on these assumptions and are robust against different model formulations. Individuals in the early latent compartment $L_{1}$ can progress either to active disease $(I)$ with rate $\phi \delta$ or to a persistent latent infection $\left(L_{2}\right)$ with rate $(1-\phi) \delta$, following the approach in Ziv et al. (2001). Parameter $\phi$ reflects that only $5 \%$ of infected individuals will ever develop active TB (Styblo, 1978; Small and Fujiwara, 2001). We choose $\delta$ such that the progression rate from early infections to active disease is $\phi \delta=0.6 \mathrm{yr}^{-1}$, which roughly approximates the data by Styblo (1978) describing the proportions of disease devel- Q2 opment after conversion. For the rates of reactivation we adopt $\omega=0.0002 \mathrm{yr}^{-1}$ for untreated latent infections (Sutherland et al., 1982; Vynnycky and Fine, 1997) and $\omega_{R}=0.00002 \mathrm{yr}^{-1}$ for those who have undergone a therapeutic intervention. As in Gomes et al. (2004a), the partial susceptibility factor affecting the rate of exogenous

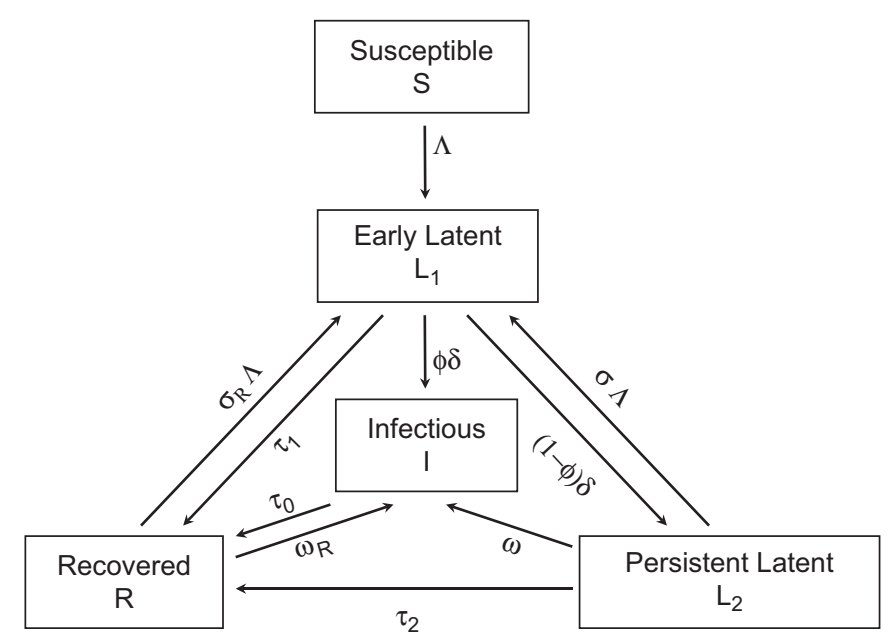

Fig. 1. Model for TB transmission with treatment of latent infections or a prophylactic vaccine. The parameters are described in Table 1. 
Table 1

Model parameters

\begin{tabular}{lll}
\hline Symbol & Definition & Value \\
\hline$\beta$ & Transmission coefficient & Variable \\
$\mu$ & Death rate and birth rate & $1 / 70 \mathrm{yr}^{-1}$ \\
$\delta$ & Rate at which individuals leave $L_{1}$ compartment & $12 \mathrm{yr}^{-1}$ \\
$\phi$ & Proportion of individuals going to compartment $I$ & 0.05 \\
$\omega$ & Rate of endogenous reactivation for persistent latent infections & $0.0002 \mathrm{yr}^{-1}$ \\
$\omega_{R}$ & Rate of endogenous reactivation for treated individuals & $0.00002 \mathrm{yr}^{-1}$ \\
$\sigma$ & Factor reducing the risk of infection as a result of acquired immunity to a previous infection for persistent & 0.25 \\
& latent individuals & \\
$\sigma_{R}$ & Factor reducing the risk of infection as a result of acquired immunity to a previous infection for treated & \\
$\tau_{0}$ & individuals & Variable \\
$\tau_{1}$ & Rate of recovery under treatment of active TB & $2 \mathrm{yr}{ }^{-1}$ \\
$\tau_{2}$ & Rate of recovery of early latent individuals under post-exposure interventions & Variable \\
\hline
\end{tabular}

reinfection of untreated individuals, $\sigma$, is fixed at 0.25 , in accordance to the highest estimates of protection conferred by BCG vaccination (Bloom and Fine, 1994). In treated patients this factor becomes $\sigma_{R}$, for which several exploratory values are adopted.

Treatment of different infection stages is implemented at specific rates: $\tau_{0}$ applies to active TB and represents the rate of recovery (typically as a result of treatment, though here it also accounts for the infrequent natural recovery); $\tau_{1}$ and $\tau_{2}$ apply, respectively, to the latent classes $L_{1}$ and $L_{2}$ as the rates at which chemotherapy or a post-exposure vaccine is applied. The rate $\tau_{0}$ is fixed at $2 \mathrm{yr}^{-1}$, corresponding to an average duration of infectiousness of 6 months, while $\tau_{1}$ and $\tau_{2}$ are considered at different exploratory values. For the rate of infection of susceptible individuals, we make the usual assumption that this is proportional to the fraction of infectious individuals, $\Lambda=\beta I$. The constant of proportionality $\beta$ is the transmission coefficient, and takes a range of values.

The basic reproduction number (Anderson and May, 1991) $R_{0}$, is proportional to the transmission coefficient, $\beta$. The relationship between the two was obtained using the approach in van den Driessche and Watmough (2002):

$R_{0}=\frac{\delta(\omega+\phi \mu)\left(\omega_{R}+\mu\right)}{\mu\left(\omega_{R}+\tau_{0}+\mu\right)(\delta+\mu)(\omega+\mu)} \beta$.

The endemic threshold $(E T)$ at $R_{0}=1$ indicates the minimal transmission potential that sustains endemic disease. The model accommodates other thresholds in transmission, namely reinfection thresholds that are central to the results presented here. The description and calculation of the endemic threshold and the reinfection threshold $(R T)$ are in the Appendix A.

\section{Interventions that reduce reactivation}

The first set of results concerns an exploration of different target subpopulations for the treatment of latent infection when the intervention does not alter the risk of reinfection. Fig. 2(a) represents the proportion of active TB individuals at equilibrium in model populations ranked by the transmission coefficient, $\beta$. The heavy line provides baseline information: it shows the proportion of individuals with active TB before treatment of latent infections is implemented $\left(\tau_{0}=2\right.$ and $\left.\tau_{1}=\tau_{2}=0\right)$. It reveals persistence of infection above the endemic threshold $\left(E T: R_{0}=1\right)$ (Anderson and May, 1991) and a steep rise in prevalence above the $R T$ ( $R T: R_{0} \approx 1 / \sigma$, as described in the Appendix A). The thinner lines show new equilibria under different strategies for treatment of latent TB. Early detection and treatment is implemented in the same fashion for all cases $\left(\tau_{0}=2\right.$ and $\left.\tau_{1}=1\right)$. The dashed lines represent the minimum and maximum effect of treating persistent latent infections, where no treatment $\left(\tau_{2}=0\right)$, and immediate treatment $\left(\tau_{2} \rightarrow \infty\right)$, respectively, gives the upper and lower bounds. For illustration, the full line corresponds to a moderate treatment rate of persistent latent infections $\left(\tau_{2}=0.1\right)$. Fig. 2(b) shows the proportional reduction that each intervention has on TB prevalence. Above the $R T$, the reduction fades out.

\section{Interventions that alter reinfection}

The second set of results emerges from relaxing the strict assumption that latent and recovered (treated) individuals have the same reinfection risk. Figs. 3 and 4 describe the results of differentiating the risks of reinfection of these two classes. Fig. 3 is obtained for illustration of the driving mechanisms, by neglecting reactivation $\left(\omega=\omega_{R}=0\right)$. This simpler context provides deeper insight, revealing the central role of reinfection on post-exposure intervention outcomes. Fig. 4 demonstrates that qualitatively equivalent results are obtained when reactivation is incorporated into the model.

Fig. 3(b) shows the prevalence of active TB in equilibrium without reactivation of persistent infections, and marks the position of the endemic $\left(R_{0}=1\right)$ and reinfection $\left(R_{0}=1 / \sigma\right)$ thresholds on the transmissibility 


\section{ARTICLE IN PRIESS}
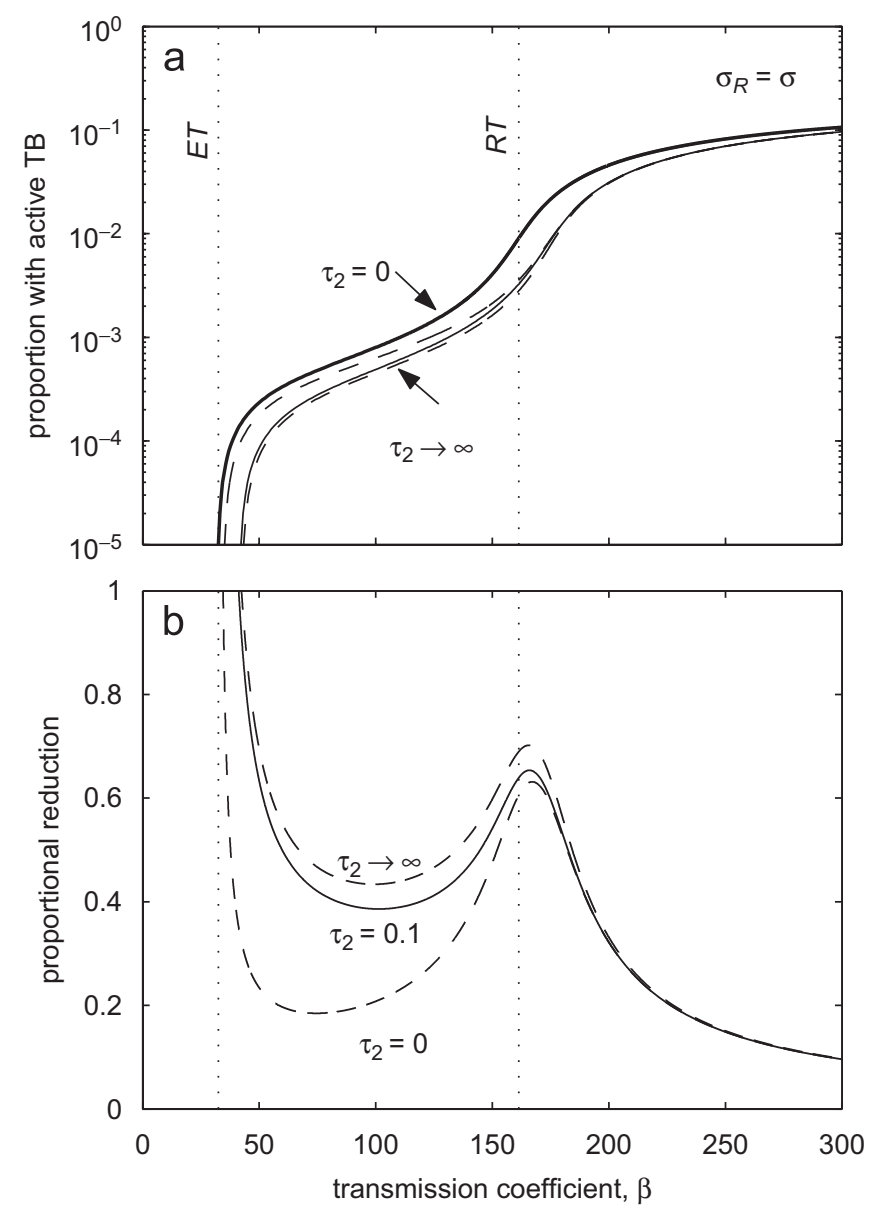

Fig. 2. Treatment of latent $\mathrm{TB}$ infection when treatment reduces reactivation rates and does not alter reinfection rates. (a) The heavy line represents the baseline proportion of active TB individuals $\left(\tau_{1}=\tau_{2}=0\right)$ and the thinner lines show lower proportions due to the implementing treatment of recent latent infections at a fixed rate $\left(\tau_{1}=0\right)$ in combination with treatment of persistent latent infections at three distinct rates: the two extremes $\left(\tau_{2}=0, \tau_{2} \rightarrow \infty\right)$ in dashed lines; and an intermediate rate $\left(\tau_{2}=0.1\right)$ in solid line. (b) Proportional reduction in prevalence attributed to the two treatment programs. The epidemic threshold $\left(R_{0}=1\right)$ and the reinfection threshold $\left(R_{0} \approx 1 / \sigma\right)$ are marked as $E T$ and $R T$, respectively.

axis $(\beta)$. This plot is equivalent to Fig. 2(a) with $\omega=\omega_{R}=0$. Two possible scenarios for the way treatment changes the risk of exogenous reinfection are explored in Figs. 3(a), (c): (a) susceptibility decreases after treatment $\left(\sigma_{R}<\sigma\right)$; (c) susceptibility increases after treatment $\left(\sigma_{R}<\sigma\right)$.

In the absence of treatment of latent infection $\left(\tau_{1}=\tau_{2}=0\right)$, the equilibrium proportion of the population that has active TB (heavy lines) is similar for all three scenarios. However, notable differences emerge when widespread treatment of latent infections is implemented (thin lines). As before, early detection and treatment is equally implemented in all cases $\left(\tau_{1}=1\right)$, and the dashed lines illustrate the limiting cases of extending the intervention to persistent infections $\left(\tau_{2}=0\right.$ and $\left.\tau_{2} \rightarrow \infty\right)$. The differentiation of reinfection risks $\left(\sigma_{R} \neq \sigma\right)$ unfolds the $R T$ into two distinct transmissibility values: $R_{0}=1 / \sigma$, in association with reinfection of individuals in the latent
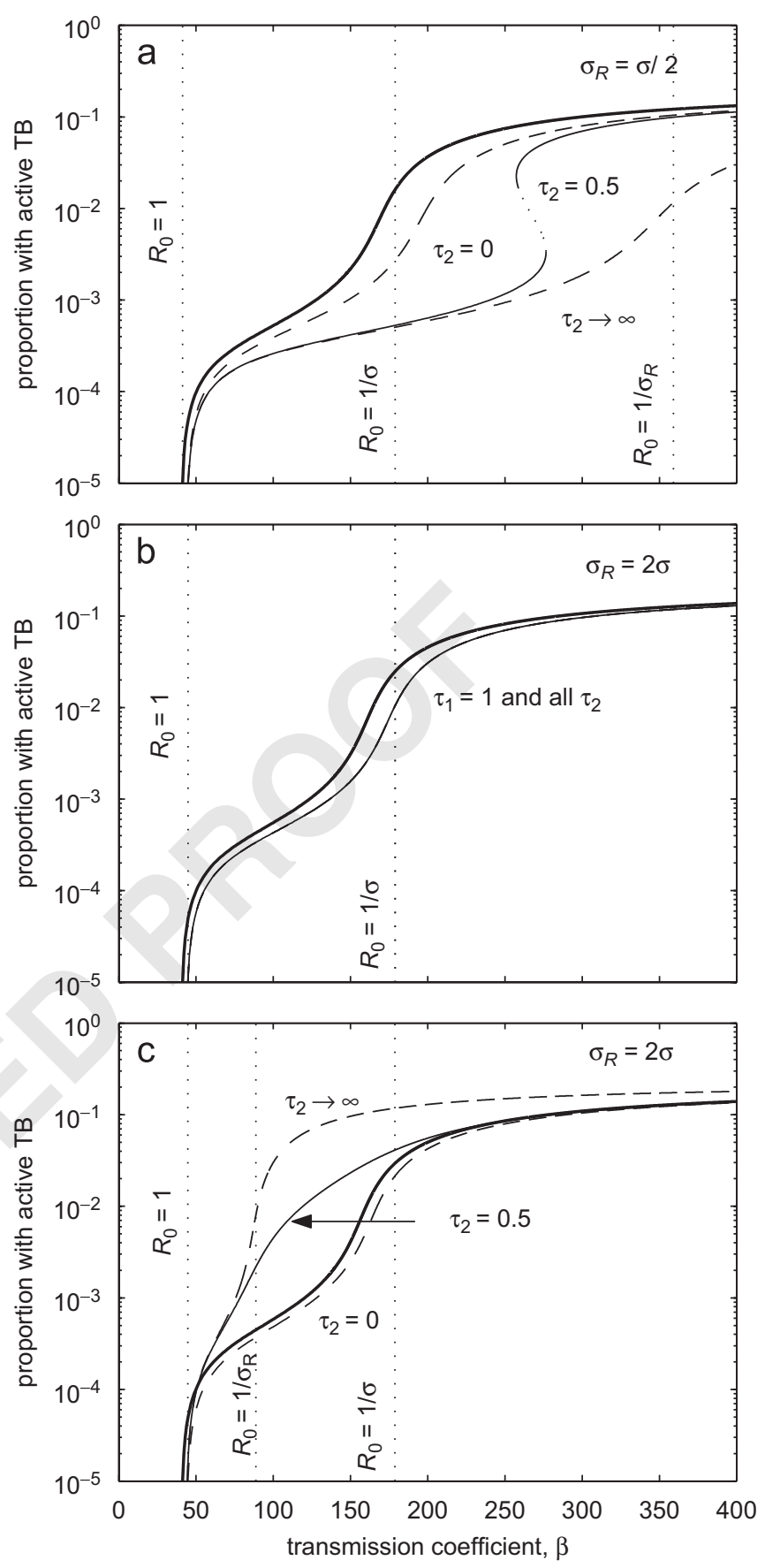

Fig. 3. Illustration of how the proportion of active TB individuals changes if the susceptibility to reinfection of treated individuals differs from that of latents: (a) $\sigma_{R}<\sigma$; (b) $\sigma_{R}=\sigma$; (c) $\sigma_{R}>\sigma$. The heavy lines represent baseline equilibria, and the thinner lines show the proportions of active TB individuals under different treatment programs. Treatment of recent converters $\left(L_{1}\right)$ is implemented at a fixed rate $\left(\tau_{1}=1\right)$, and treatment of persistent latent infections $\left(L_{2}\right)$ is implemented at the indicated rates $\left(\tau_{2}\right)$. The plots show equilibrium proportions of active TB under the simplifying assumption that reactivations do not occur, $\omega=\omega_{R}=0$.

class $\left(L_{2}\right)$; and $R_{0}=1 / \sigma_{R}$, in association with reinfection of individuals in the treated class $(R)$. These transmission rates bound the parameter region where post-exposure treatment regimes have most impact. An important finding is that the two boundaries appear in reverse order when 


\section{ARTICLE IN PRESS}
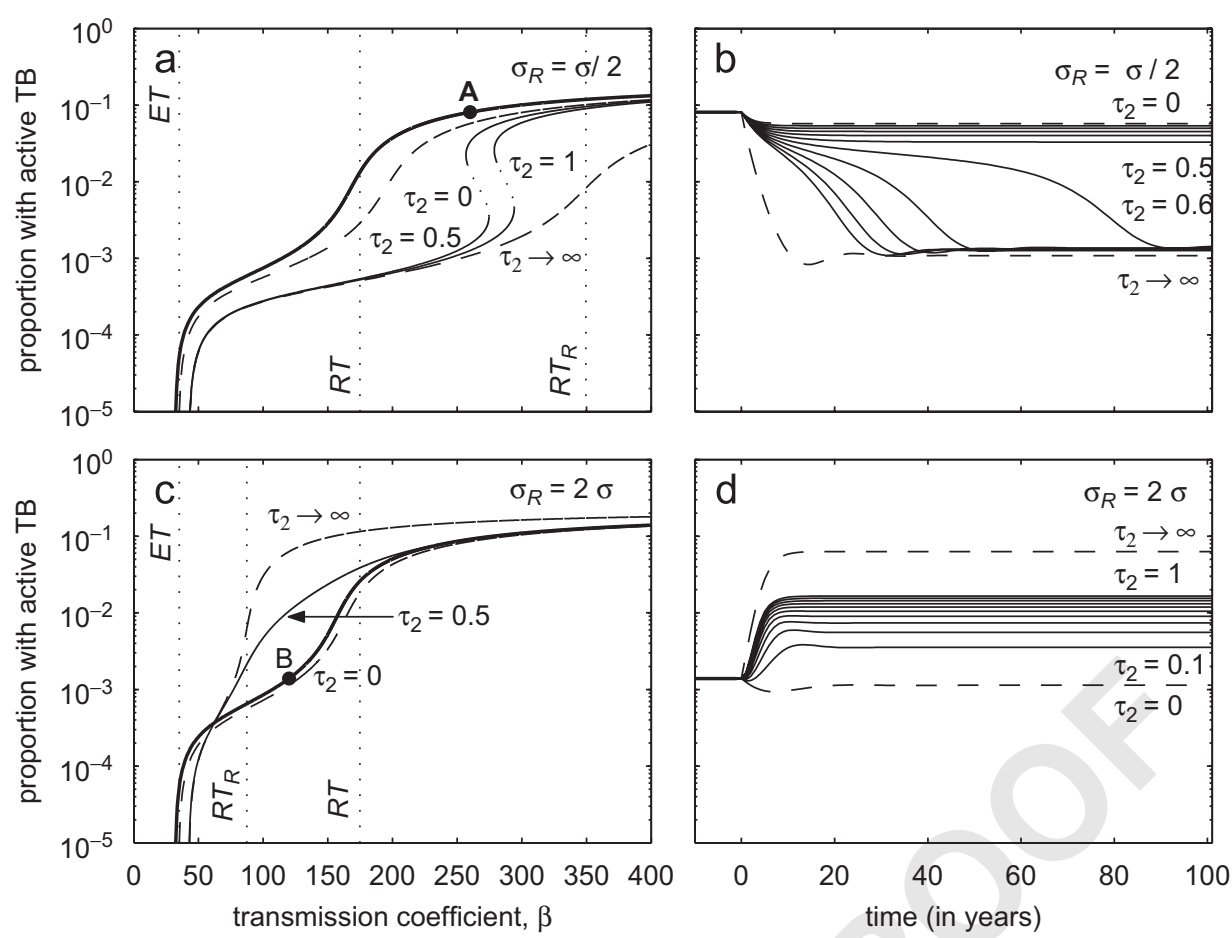

Fig. 4. Illustration of how the proportion of active TB individuals changes if the susceptibility of treated individuals differs from that of latents: (a, c) show equilibrium proportions of active TB; $(b, d)$ show simulations following the proportion of active TB individuals over time since the introduction of treatment of latent infections. The heavy lines represent baseline equilibria, and the thinner lines show the proportions of active TB individuals under different treatment programs. Treatment of recent converters $\left(L_{1}\right)$ is implemented at a fixed rate $\left(\tau_{1}=1\right)$, and treatment of persistent latent infections $\left(L_{2}\right)$ is implemented at a series of rates $\left(\tau_{2}=0 ; 01 ; 0.2 ; \ldots ; 1\right.$; and the limit $\left.\tau_{2} \rightarrow \infty\right)$. Epidemic and reinfection thresholds are marked by $E T, R T$ and $R T_{R}$.

$\sigma_{R}<\sigma$ (Fig. 3(a)) and $\sigma_{R}>\sigma$ (Fig. 3(c)). As detailed next, boundary order underlies dynamic changes that have crucial implications for public health.

The outcome of treating latent TB at a moderate rate lies between the two incidence levels observed at the upper and lower transmission boundaries described above. However, the relative magnitude of $\sigma$ and $\sigma_{R}$ determines whether the intervention will cause a reduction or an increase in the prevalence of active TB. The intermediate outcomes are illustrated by considering the effect of $\tau_{2}=0.5$ in the two possible scenarios. In the first scenario $\left(\sigma_{R}<\sigma\right)$, the intervention is always beneficial, as the treated class increases the effective reinfection threshold. The prevalence of active TB decreases due to the reduction in susceptibility to reinfection. Moreover, the thin lines now reveal an $S$ shaped form in the relation between transmission and prevalence of active TB (Fig. 3(a)), accommodating bistable equilibria with basins of attraction that are separated by an unstable equilibrium (dotted segment). The intervention outcome is remarkably different if susceptibility to reinfection increases after treatment $\left(\sigma_{R}>\sigma\right)$. In this case, there is a range of transmissibilities where the thin lines lie above the heavy line, implying an increase in active TB prevalence in the population due to the implementation of widespread treatment of persistent latent TB. This occurs because the $R T$ associated with the treated class is lower.

\section{Interventions that alter reactivation and reinfection}

Here we review the analyses when reactivation is included in the model. Expressions for the corresponding reinfection thresholds are given by equalities (A.4) and (A.6) in the Appendix A, but are approximated by $R_{0}=1 /$ $\sigma$ and $R_{0}=1 / \sigma_{R}$ when $\omega$ and $\omega_{R}$ are small (here, $\omega=0.0002$ and $\left.\omega_{R}=0.00002\right)$. Precise threshold values, marked $R T$ and $R T_{R}$, appear in Figs. 4(a), (c), in plots qualitatively equivalent to Figs. 3(a), (c). Figs. 4(b), (d), trace model dynamics in time upon intervention implementation over a range of rates $\tau_{2}$, illustrating the bistable behavior associated with the $S$-shaped curves in Figs. 3(a) and 4(a).

We use initial conditions corresponding to the endemic infectious level marked by point A in Fig. 4(a) to show how intervention efforts modify model dynamics under the first scenario $\left(\sigma_{R}<\sigma\right)$. Fig. 4(b) shows the prevalence of active TB over time upon introducing treatment of latent infection at rates $\tau_{2}=0.1,0.2, \ldots, 1.0$. Dashed lines mark the limiting cases, $\tau_{2}=0$ and $\tau_{2} \rightarrow \infty$. A critical treatment rate of $\tau_{2} \sim 0.5$ separates policies of contrasting success. The reduction in prevalence is small to moderate for $\tau_{2} \leqslant 0.5$ (sub-critical intervention) and very large for $\tau_{2} \geqslant 0.6$ (supercritical intervention). Bistable dynamics have been described previously for hepatitis-B (Medley et al. 2001), in host-parasite interactions (May, 1977) and in various ecosystems (Scheffer et al., 2001). The potential for 


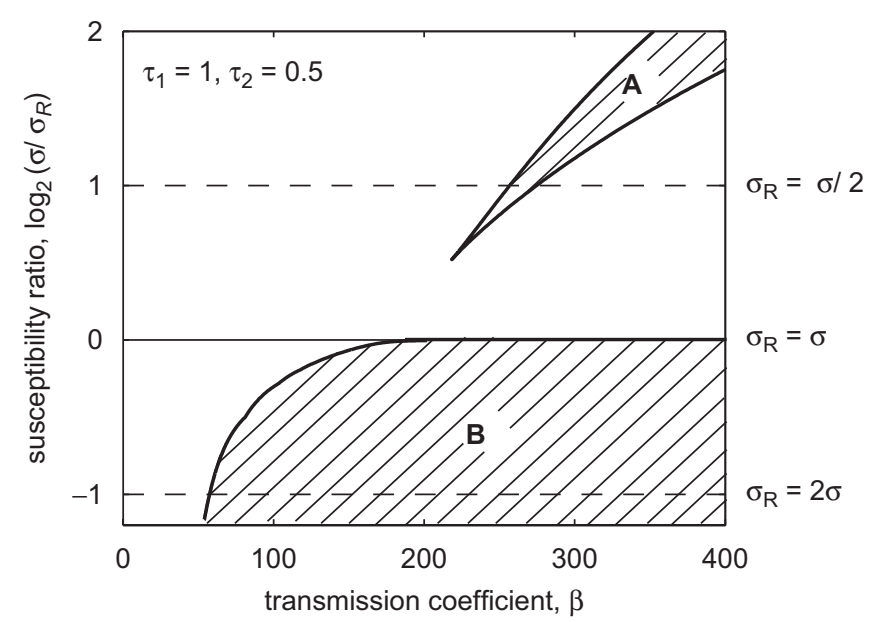

Fig. 5. Consequences of post-exposure treatment summarized in a twoparameter diagram. The shaded regions mark the new model behavior when widespread treatment is implemented at the rates $\tau_{1}=1, \tau_{2}=0.5$ : bistability (marked A); increased prevalence (marked B) and illustrated by scenarios A and B, respectively, in Fig. 4.

dramatic changes characteristic of bistability must be taken into account when evaluating both conservation and eradication strategies.

To simulate the second scenario $\left(\sigma_{R}>\sigma\right)$ we use model population B (marked in Fig. 4(c)). The associated dynamics are represented in Fig. 4(d). In this case, the order of two reinfection thresholds does not lead to bistability, but widespread treatment of latent infection leads to an increase in prevalence of active TB for a range of transmissibilities. Whether the effect of the intervention is positive or negative depends on the balance between the decreased risk of endogenous reactivation and the increased risk of exogenous reinfection (Fig. 4(c)).

Fig. 5 provides a summary of the behavior attributed to differences in susceptibility to reinfection between persistent latent and recovered individuals. The horizontal line at zero corresponds to no difference in susceptibility $\left(\sigma_{R}=\sigma\right)$. Above the line, recovered individuals are less susceptible $\left(\sigma_{R}<\sigma\right)$ and bistability occurs in the shaded area (marked A) with the typical shape of a cusp catastrophe (Zeeman, 1977). Below the line, recovered individuals are more susceptible $\left(\sigma_{R}>\sigma\right)$ and the shaded area (marked B) indicates the region where widespread treatment of latent TB leads to an increase in disease prevalence. These effects are triggered by a widespread post-exposure intervention.

\section{Bistability and catastrophic dynamics}

The interplay between population compartments with different susceptibility status is what gives rise to bistability: a factor $\sigma$ modifies susceptibility of individuals who harbor untreated persistent latent infections $\left(L_{2}\right)$; and a different factor $\sigma_{R}$ affects susceptibility of those who have been treated $(R)$. In the absence of treatment of persistent latent infection $\left(\tau_{2}=0\right)$ the prevalence of active TB at equilibrium undergoes a steep increase at the $R T$. A widespread strategy of treatment of latent infection (high $\tau_{2}$ ) has the potential to bring the endemic equilibrium to a curve whose $R T$ is $R T_{R}$. Intervention impact can be unexpectedly high for populations whose transmissibility lies between $R T$ and $R T_{R}$. When $R T_{R}$ is greater than $R T$ (i.e. $\left.\sigma_{R}<\sigma\right)$ this potential represents dramatic benefits to TB control, but whether it will be made real depends critically on intervention design.

The dynamics exhibited here include two alternative ways out of $L_{2}$ with opposite effects on the prevalence of disease: natural progression of infection increases the incidence of active TB (via reactivation or reinfection, $\omega+\sigma \beta I)$; and human interventions are designed to reduce the incidence of active TB (via treatment, $\tau_{2}$ ). As such, natural processes and medical interventions can be seen as competing for individuals in $L_{2}$. The bistable outcome of the intervention is explained by noting that the first process increases with the prevalence of active $\mathrm{TB}$, while the second is constant. It is therefore not surprising that a threshold in the prevalence of active TB dictates whether the intervention "wins" or "loses" over natural progression. While the prevalence of active TB remains above this threshold, natural processes causing disease are the winners and the intervention has little impact. If the prevalence drops below the threshold, the intervention wins and succeeds in bringing active TB close to the lower equilibrium curve. Fig. 4(b) reflects this phenomenon at intermediate rates of treatment of latent TB. Initially, treatment of latent infections induces only a slow decrease in the prevalence of active TB. Large numbers of untreated latent individuals reacquire infection at the relatively fast rate $\sigma \beta I$, thus slowing down disease control. However, once treatment reduces availability of persistent latent individuals below a threshold (at the unstable equilibrium), a more rapid decrease in the prevalence of active TB follows. From this point onwards, most reinfections occur in treated individuals at a comparatively lower rate $\sigma_{R} \beta I$. This change in behavior can be explored in the interest of control by appropriately varying the treatment rate during the course of the intervention.

\section{Consequences to intervention design}

Fig. 6 illustrates the potential of interventions that are implemented at high rates during an initial phase, relaxing them later to lower rates. Starting from equilibrium A (as in Fig. 4(a)), alternative intervention strategies are initiated at time $t=0$. The duration of the initial phase is critical to intervention outcome: the higher line traces the time course of active TB if $\tau_{2}=1$ is maintained for 13 years, and the lower line shows the result of extending this initial (more intense) phase to 14 years. In both cases, the intervention is then relaxed to $\tau_{2}=0.5$. Contrasting prevalence levels result, demonstrating the way in which similar public health measures can have radically different long-term effects on disease control owing to bistability. This sensitivity is a characteristic of catastrophic dynamics 


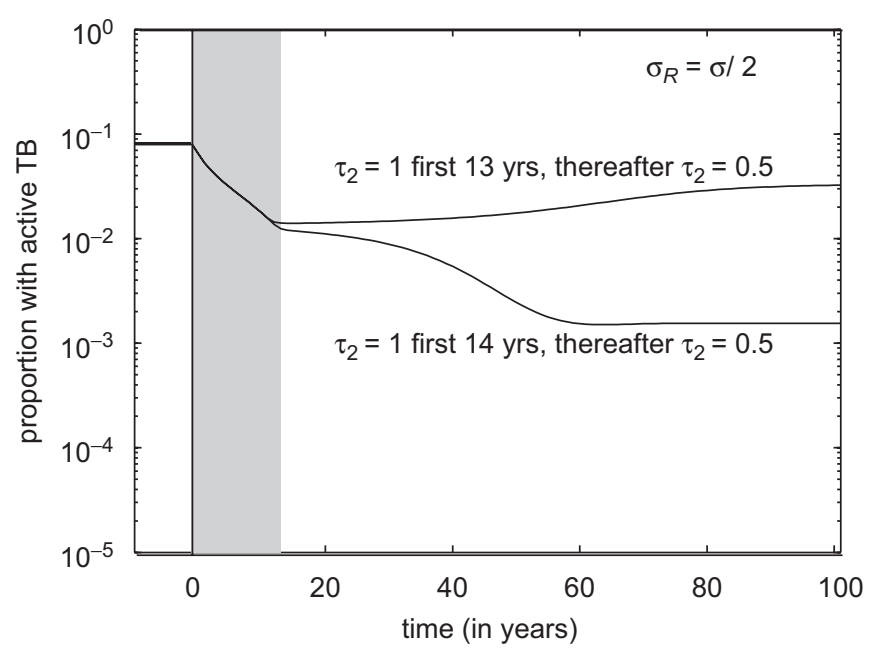

Fig. 6. Simulations following the proportion of active TB individuals over time since the introduction of widespread treatment of latent infections. The treatment rate is initially set at $\tau_{2}=1$ (shaded band) and later relaxed to $\tau_{2}=0.5$ (non-shaded area to the right). The figure illustrates that a shift from the higher to the lower equilibrium branch occurs when the higher treatment rate $\tau_{2}=1$ is prolonged between 13 and 14 years.

(Zeeman, 1977) and has dramatic implications for public health. Similar bistable dynamics have also been predicted by previous TB models (Feng et al., 2000; Singer and Kirschner, 2004), but relied on arguably unrealistic parameter assumptions (Lipsitch and Murray, 2003).

\section{Discussion}

In this paper, mathematical models were used to help define target subpopulations for screening and treating latent $\mathrm{TB}$ infections, and to explore the potential of widespread post-exposure interventions such as chemotherapy or post-exposure vaccination. The primary requirement for such medical interventions is the capacity to reduce the risk of reactivation of latent infections. Since the risk of progression to active TB is especially high during the initial years following infection, recent converters should definitely be treated. As for the adoption of more widespread interventions, our results are more elaborate, and depend critically on whether the intervention decreases or increases susceptibility to reinfection. Given the lack of published studies providing direct evidence for either assumption, we explored both possibilities and described their public health implications. Indeed, on the one hand one could argue that latent infection helps to sustain immunity to tuberculosis. In this case, treatment would increase the risk that subsequent infection leads to active TB. On the other hand, the risk of reactivation may be higher in untreated hosts, who harbor larger amounts of bacteria than those treated. Reinfection may then trigger reactivation of a latent infection (Warren et al., 2004), which suggests that treatment would reduce the risk of reinfection.
According to our model, the scenario where treatment reduces the risk of reinfection gives rise to conditions of bistability: the impact of the intervention depends critically on whether the treatment rate is above or below a threshold. If the treatment rate is increased across this critical value, the resulting reductions in TB-prevalence in regions of high burden of disease go up by one or two orders of magnitude. As is typical for such catastrophic dynamics, the existence of bistability is unapparent and has no "early-warning signals" until a tiny change in treatment rate triggers a large shift from one dynamical regime to the other (Zeeman, 1977).

If, on the contrary, treatment increases the risk of reinfection, it will be of limited benefit. Success depends then on the balance between decreasing reactivation and increasing reinfection rates, which likely will vary among populations.

These contrasting results evidence how reinfection may fundamentally affect control strategies, and underscore the need to understand the host-pathogen interaction mechanisms that determine reinfection risk in $M$. tuberculosis. Epidemiologic study designs with the capacity to separately estimate the impact of post-exposure interventions on reactivation and reinfection would therefore be decisive.

The qualitative effects presented in this paper are generally robust against reasonable changes in parameter values and model assumptions. The results persist the inclusion of TB-related death rates and varying population size as well as the implementation of different demographics (with constant recruitments and linear death terms as in Murphy et al., 2002, 2003) and a wide range of reactivation rates (these results will be presented elsewhere). In fact, reactivation parameters $\omega$ and $\omega_{R}$ were set to zero in Section 4 of this paper for illustration purposes, without provoking any significant change in the predicted behavior. Furthermore, the transmissibility parameter $\beta$ has been abundantly explored in bifurcation diagrams, and parameters corresponding to treatment rates, $\tau_{0}, \tau_{1}$ and $\tau_{2}$, being the object of study, were varied as appropriate. On the other hand, parameter values for $\delta$ and $\phi$ have been taken from well-accepted estimates in the literature and can be varied within reasonable ranges without changing the reported effects qualitatively. Partial susceptibility factors $\sigma$ and $\sigma_{R}$, that affect reinfection rates, are crucial to determine the position of the behavior of interest (i.e., reinfection thresholds) on the transmissibility axis. Increasing (decreasing) one of these parameters shifts the associated $R T$ to the left (right) according to Eqs. (A.4) and (A.6) in the Appendix A. Hence, the observed effects can also occur in smaller as well as larger ranges of transmission coefficients (and, equivalently, $R_{0}$ ).

One aspect that deserves further exploration, however, is the sensitivity of the results on the total rate of departure from the early latent class, $\delta$. It should be noted that the bistable behavior reported here is observed when this rate is larger than around $4 \mathrm{yr}^{-1}$, corresponding to an average time of 3 months or less before an individual moves to 
1 persistent latent or active TB. The majority of mathematical models in the TB literature, however, bypass this state by implementing direct transitions from susceptible states to either persistent latent or active TB, corresponding to an infinitely large $\delta$ (see, for example, Blower et al. 1995; Dye Q3 et al., 1998; Feng et al. 2000; Gomes et al. 2004a; Murphy 7 et al. 2002, 2003; Murray and Salomon, 1998). The implicit representation of an early latent class has been exceptionally implemented in few models (Aparicio et al., 2002; Ziv et al., 2001; Vynnycky and Fine, 1997). Vynnycky and Fine (1997) use a value of $0.5 \mathrm{yr}^{-1}$, while Ziv et al. (2001) of $1.5 \mathrm{yr}^{-1}$ and Aparicio et al. (2002) a time-dependent value that is always larger than $1 \mathrm{yr}^{-1}$. Parameter choices can depend critically on the context of the particular model structure. To our knowledge, there are no data unequivocally suggesting a certain parameter range. This holds in particular for the progression rate $\left(1-\phi \delta\right.$ from early $\left(L_{1}\right)$ to persistent latent infections $\left(L_{2}\right)$, whereas we have a good motivation for the parameter value of the rate $\phi \delta$ from early latent infections to active disease $(I)$. Future extensions might consider a relaxation of this latter rate.

Finally, the model presented here extends earlier models (Blower et al., 1995; Feng et al., 2000; Gomes et al., 2004a; Lietman and Blower, 2000; Murphy et al., 2002, 2003; Singer and Kirschner, 2004; Ziv et al., 2001, 2004), but tailors more complicated models (Murray and Salomon, 1998), in order to gain insight into the underlying dynamics (Anderson, 1998). The effects presented do not rely on the complexities of reactivation that characterize tuberculosis, and may be applicable to other diseases where natural infection and medical interventions alter the risk of (re)infection upon subsequent exposures.

\section{Acknowledgments}

We thank Manuel Gomes, Carlota Rebelo, Herbert Hethcote and one reviewer (Juan Aparicio) for valuable comments. This research was funded by the Gulbenkian Foundation (FCG), the Portuguese Research Council (FCT) and the European Commission, grant MEXT-CT2004-14338.

\section{Appendix A}

\section{A1. Interpretation of $R_{0}$}

The expression of $R_{0}$, given by (2) in Section 2,

$R_{0}=\frac{\delta(\omega+\phi \mu)\left(\omega_{R}+\mu\right)}{\mu\left(\omega_{R}+\tau_{0}+\mu\right)(\delta+\mu)(\omega+\mu)} \beta$

can be written as the sum of different progressions into active TB. In the simpler case $\omega_{R}=0$ we can write

$R_{0}^{*}=\frac{\beta}{\left(\tau_{0}+\mu\right)}\left[\frac{\phi \delta}{(\delta+\mu)}+\frac{(1-\phi) \delta}{(\delta+\mu)} \frac{\omega}{(\omega+\mu)}\right]$.

The first factor in Eq. (A.1) gives the average number of secondary cases produced by a single individual with active
$\mathrm{TB}$ in a completely susceptible population during its infectious period. The first and second terms in the second factor reflect that individuals progress towards active TB through either the early latent and persistent latent class, respectively. When $\omega_{R}>0$, recovered individuals have an extra opportunity to progress to active TB that does not depend on re-exposures. This extra contribution for the infectious period is expressed by the term

$$
\begin{aligned}
\frac{\left(\tau_{0}+\mu\right)\left(\omega_{R}+\mu\right)}{\mu\left(\omega_{R}+\tau_{0}+\mu\right)}= & \frac{1}{1-\tau_{0} \omega_{R} /\left(\tau_{0}+\mu\right)\left(\omega_{R}+\mu\right)} \\
= & 1+\frac{\tau_{0}}{\left(\tau_{0}+\mu\right)} \frac{\omega_{R}}{\left(\omega_{R}+\mu\right)} \\
& +\left(\frac{\tau_{0}}{\left(\tau_{0}+\mu\right)} \frac{\omega_{R}}{\left(\omega_{R}+\mu\right)}\right)^{2}+\ldots,
\end{aligned}
$$

that results from the countless opportunities the infected individual has to repeat this event. The full expression for $R_{0}$ is then the product of Eqs. (A.1) and (A.2).

\section{A2. Calculation of the reinfection threshold}

Reinfection thresholds correspond to critical transmissibility values, above which there is a steep nonlinear increase in disease prevalence. This is because infection can be maintained in a population of previously infected (and partially immune) individuals. These thresholds are approximated using reinfection submodels that isolate reinfection from other mechanisms (such as primary infection and reactivation). Such submodels are obtained by considering a population where all individuals have been challenged previously, thus acquiring a degree of immunity, and by assuming that reactivation is absent $\left(\omega=\omega_{R}=0\right)$. A bifurcation in the submodels is then associated to an $R T$ in the full model (Gomes et al., 2004b, 2005; Breban and Blower, 2005).

The case $\sigma_{R}=\sigma$ leads to a system where the classes $L_{2}$ and $R$ are equivalent and can be merged. The reinfection submodel supports endemic equilibria when transmission is above the critical value

$\beta=\frac{1}{\sigma} \frac{\left(\tau_{0}+\mu\right)\left(\delta+\tau_{1}+\mu\right)}{\delta \phi}$,

hereafter called the reinfection threshold $(R T)$. Substituting Eq. (2) into Eq. (A.1), the $R T$ is written in terms of $R_{0}$ as

$R_{0}=\frac{1}{\sigma} \frac{\delta(\omega+\phi \mu)\left(\omega_{R}+\mu\right)\left(\tau_{0}+\mu\right)\left(\delta+\tau_{1}+\mu\right)}{\delta \phi \mu\left(\omega_{R}+\tau_{0}+\mu\right)(\delta+\mu)(\omega+\mu)}$.

The simpler relation $R_{0}=1 / \sigma$ (Gomes et al., 2004a, b) is obtained for the particular case $\omega=\omega_{R}=0$ and no intervention is in place $\left(\tau_{1}=0\right)$.

For the case $\sigma_{R} \neq \sigma$, we consider two extreme scenarios for the treatment of persistent latent infections: $\tau_{2}=0$-no treatment of persistent infections; and $\tau_{2} \rightarrow \infty$-idealized instantaneous treatment.

When post-exposure measures apply only to early detection $\left(\tau_{2}=0\right)$, the dynamics of the full model are 
1 determined by the reinfection in the latent class $L_{2}$. The reinfection submodel can then be seen to support an endemic equilibrium when transmission is above the reinfection threshold (A.3).

If, on the other extreme, we consider that latent individuals move instantaneously out of class $L_{2}$ into the recovered class $R$ (by treatment of persistent latents at rate $\left.\tau_{2} \rightarrow \infty\right)$, the dynamics are determined by reinfection of the recovered class. It is therefore assumed in the reinfection submodel, that all individuals entering the system go into the $R$ class, where they are subject to the factor of protection, $\sigma_{R}$. Reinfection of recovered individuals may then sustain an endemic equilibrium if transmission is above the alternative reinfection threshold $\left(R T_{R}\right)$

$\beta=\frac{1}{\sigma_{R}} \frac{\left(\tau_{0}+\mu\right)\left(\delta+\tau_{1}+\mu\right)}{\delta \phi}$,

which can be written in terms of $R_{0}$ as

$R_{0}=\frac{1}{\sigma_{R}} \frac{\delta(\omega+\phi \mu)\left(\omega_{R}+\mu\right)\left(\tau_{0}+\mu\right)\left(\delta+\tau_{1}+\mu\right)}{\delta \phi \mu\left(\omega_{R}+\tau_{0}+\mu\right)(\delta+\mu)(\omega+\mu)}$.

Transmission thresholds $E T, R T$ and $R T_{R}$, associated with this model are marked as vertical dotted lines in Figs. $2-4$.

\section{References}

Anderson, R.M., 1998. Tuberculosis: old problems and new approaches. Proc. Natl. Acad. Sci. USA 95, 13352-13354.

Anderson, R.M., May, R.M., 1991. Infectious Diseases of Humans: Dynamics and Control. Oxford University Press, Oxford, 757pp.

Andries, K., Verhasselt, P., Guillemont, J., Göhlmann, H.W.H., Neefs, J.M., et al., 2005. A diarylquinoline drug active on the ATP synthase of Mycobacterium tuberculosis. Science 307, 223-227.

Aparicio, J.P., Capurro, A.F., Castillo-Chavez, C., 2002. Markers of disease evolution: the case of tuberculosis. J. Theor. Biol. 215, 227-237.

Bandera, A., Gori, A., Catozzi, L., Degli Esposti, A., Marchetti, G., et al., 2001. Molecular epidemiology study of exogenous reinfection in an area with a low incidence of tuberculosis. J. Clin. Microbiol. 39, 2213-2218.

Bloom, B.R., Fine, P.E., 1994. The BCG experience: implications for future vaccines against tuberculosis. In: Bloom, B.R. (Ed.), Tuberculosis: Pathogenesis, Protection and Control. American Society for Microbiology, Washington, DC, pp. 531-552.

Bloom, B.R., McKinney, J.D., 1999. The death and resurrection of tuberculosis: DNA vaccines may be useful in overcoming persistent tuberculosis infections. Nat. Med. 5, 872-874.

Blower, S.M., McLean, A.R., Porco, T.C., Small, P.M., Hopewell, P.C., et al., 1995. The intrinsic transmission dynamics of tuberculosis epidemics. Nat. Med. 1, 815-821.

Borgdorff, M.W., Floyd, K., Broekmans, J.F., 2002. Interventions to reduce tuberculosis mortality ant transmission in low and middleincome countries. Bull. WHO 80, 217-227.

Breban, R., Blower, S., 2005. The reinfection threshold does not exist. J. Theor. Biol. 235, 151-152.

Caminero, J.A., Pena, M.J., Campos-Herrero, M.I., Rodriguez, J.C., Afonso, O., et al., 2001. Exogenous reinfection with tuberculosis on a European island with a moderate incidence of disease. Am. J. Respir. Crit. Care Med. 163, 717-720.

Chiang, C.Y., Riley, L.W., 2005. Exogenous reinfection in tuberculosis. Lancet Infect. Dis. 5, 629-636.
Dye, C., Garnett, G.P., Sleeman, K., Williams, B.G., 1998. Prospects for worldwide tuberculosis control under the WHO DOTS strategy. Lancet 352, 1886-1891.

Feng, Z., Castillo-Chavez, C., Capurro, A.F., 2000. A model for tuberculosis with exogenous reinfection. Theor. Pop. Biol. 57, 235-247.

Gomes, M.G.M., Franco, A.O., Gomes, M.C., Medley, G.F., 2004a. The reinfection threshold promotes variability in tuberculosis epidemiology and vaccine efficacy. Proc. R. Soc. London B 271, 617-623.

Gomes, M.G.M., White, L.J., Medley, G.F., 2004b. Infection, reinfection, and vaccination under suboptimal immune protection: epidemiological perspectives. J. Theor. Biol. 228, 539-549.

Gomes, M.G.M., White, L.J., Medley, G.F., 2005. The reinfection threshold. J. Theor. Biol. 236, 111-113.

Ha, S.J., Jeon, B.Y., Kim, S.C., Kim, D.J., Song, M.K., et al., 2003. Therapeutic effect of DNA vaccines combined with chemotherapy in a latent infection model after aerosol infection of mice with Mycobacterium tuberculosis. Genet. Ther. 10, 1592-1599.

Ha, S.J., Jeon, B.Y., Youn, J.I., Kim, S.C., Cho, S.N., et al., 2005. Protective effect of DNA vaccine during chemotherapy on reactivation and reinfection of Mycobacterium tuberculosis. Genet. Ther. 12, 634-638.

Lietman, T., Blower, S.M., 2000. Potential impact of tuberculosis vaccines as epidemic control agents. Clin. Infect. Dis. 30, S316-S322.

Lipsitch, M., Murray, M.B., 2003. Multiple equilibria: tuberculosis transmission require unrealistic assumptions. Theor. Pop. Biol. 63, 169-170.

Lowrie, D.B., Tascon, R.E., Bonato, V.L.D., Lima, V.M.F., Facciolo, L.H., et al., 1999. Therapy of tuberculosis in mice by DNA vaccination. Nature 400, 269-271.

Malowany, J.I., McCormick, S., Santosuosso, M., Zhang, X., Aoki, N., et al., 2006. Development of cell-based tuberculosis vaccines: genetically modified dendritic cell vaccine is a much more potent activator of CD4 and CD8 T cells than peptide- or protein-loaded counterparts. Mol. Ther. 13, 766-775.

May, R.M., 1977. Thresholds and breakpoints in ecosystems with a multiplicity of stable states. Nature 269, 471-477.

McShane, H., Pathan, A.A., Sander, C.R., Keating, S.M., Gilbert, S.C., et al., 2004. Recombinant modified vaccinia virus Ankara expressing antigen $85 \mathrm{~A}$ boosts BCG-primed and naturally acquired antimycobacterial immunity in humans. Nat. Med. 1, 1240-1244.

Medley, G.F., Lindop, N.A., Edmunds, W.J., Nokes, D.J., 2001. Hepatitis-B virus endemicity: heterogeneity, catastrophic dynamics and control. Nat. Med. 7, 619-624.

Murphy, B.M., Singer, B.H., Anderson, S., Kirschner, D., 2002. Comparing epidemic tuberculosis in demographic distinct heterogeneous populations. Math. Biosci. 180, 161-185.

Murphy, B.M., Singer, B.H., Kirschner, D., 2003. On treatment of tuberculosis in heterogeneous populations. J. Theor. Biol. 223, 391-404.

Murray, C.J.L., Salomon, J.A., 1998. Modeling the impact of global tuberculosis control strategies. Proc. Natl. Acad. Sci. USA 95, 13881-13886.

Scheffer, M., Carpenter, S., Foley, J.A., Folke, C., Walker, B., 2001. Catastrophic shifts in ecosystems. Nature 413, 591-596.

Singer, B.H., Kirschner, D., 2004. Influence of backward bifurcation on interpretation of $R_{0}$ in a model of epidemic tuberculosis with reinfection. Math. Biosci. Eng. 1, 81-93.

Small, P.M., Fujiwara, P.I., 2001. Management of tuberculosis in the United States. N. Engl. J. Med. 345, 189-200.

Styblo, K., 1978. State of the art: epidemiology of tuberculosis. Bull. Int. Union Tuberc. 53, 141-152.

Sutherland, I., Švandová, E., Radhakrishna, S.E., 1982. The development of clinical tuberculosis following infection with tubercle bacilli. Tubercle 62, 255-268.

van den Driessche, P., Watmough, J., 2002. Reproduction numbers and sub-threshold endemic equilibria for compartmental models of disease transmission. Math. Biosci. 180, 29-48. 


\section{ARTICLE IN PRESS}

van Rie, A., Warren, R., Richardson, M., Victor, T.C., Gie, R.P., et al., 1999. Exogenous reinfection as a cause of recurrent tuberculosis after curative treatment. N. Engl. J. Med. 341, 1174-1179.

Vynnycky, E., Fine, P.E.M., 1997. The natural history of tuberculosis: the implications of age-dependent risks of disease and the role of reinfection. Epidemiol. Infect. 119, 183-201.

Warren, R.M., Victor, T.C., Streicher, E.M., Richardson, M., Beyers, N., et al., 2004. Patients with active tuberculosis often have different strains in the same sputum specimen. Am. J. Respir. Crit. Care Med. $169,610-614$.

Zeeman, E.C., 1977. Catastrophe Theory: Selected Papers. London, Addison-Wesley, 674pp.

Ziv, E., Daley, C.L., Blower, S.M., 2001. Early therapy for latent tuberculosis infection. Am. J. Epidem. 153, 381-385.

Ziv, E., Daley, C.L., Blower, S., 2004. Potential public health impact of new tuberculosis vaccines. Emerg. Infect. Dis. 10, 1529-1535. 